\title{
Very Early In-Utero Diagnosis of Walker-Warburg Phenotype: The Cutting Edge of Technology
}

\author{
Authors \\ R. Achiron ${ }^{1}$, E. Katorza ${ }^{2}$, H. Reznik-Wolf ${ }^{3}$, E. Pras ${ }^{3}$, D. Kidron ${ }^{4}$, M. Berkenstadtt ${ }^{2}$ \\ Affiliations \\ ${ }^{1}$ Obstetrics and Gynecology, Sheba Medical Center Tel-Hashomer, Tel-Aviv, Ramat Gan, Israel \\ 2 Obstetrics and Gynecology, Sheba Medical Center, Ramat Gan, Israel \\ ${ }^{3}$ Institute of Human Genetics, Sheba Medical Center, Ramat-Gan, Israel \\ ${ }^{4}$ Institute of Pathology, Sheba Medical Center, Ramat-Gan, Israel
}

Key words

- walker-warburg phenotype

- prenatal diagnosis

- advanced technology

- transvaginal ultrasound

- ultrasound

- obstetrics

\begin{abstract}
$\nabla$

Background: Walker-Warburg phenotype is a severe and lethal autosomal recessive disorder, belonging to a group of congenital malformations defined as abnormal pial basement membrane formation. So far, prenatal diagnosis was considered possible only during late pregnancy. Methods: First trimester assessment of a pregnancy suspected to be affected by WalkerWarburg phenotype, using a high-resolution transvaginal ultrasound probe $(6-12 \mathrm{MHz}), \mathrm{T} 2$ MR imaging (1.5T), molecular genetics and histopathology.
\end{abstract}

Results: Very early diagnosis of the WalkerWarburg phenotype at 11 weeks of gestation proved possible by depicting the classic signs of this entity, confirmed by molecular genetics, post-abortion MR imaging and histopathology.

Conclusion: Advancements in ultrasound equipment and technology, molecular genetics and histopathology have made very early detection of this syndrome possible, thus shedding new light on the natural history of this malformation.

\section{received $\quad 11.06 .2015$ \\ accepted $\quad 12.10 .2015$}

\section{Bibliography}

DOI http://dx.doi.org/

10.1055/s-0036-1582303

Ultrasound International Open

2016; 2: E54-E57

(c) Georg Thieme Verlag KG

Stuttgart - New York

ISSN 2199-7152

Correspondence

Dr. E. Katorza

Obstetrics and Gynecology

Sheba Medical Center

Ramat Gan

Israel 52621

Tel.: + 972/3/5308 116

eldad.katorza@gmail.com

\section{License terms}

\section{Introduction}

$\nabla$

The Walker-Warburg phenotype belongs to a group of congenital malformations recently defined as malformation due to abnormal pial basement membrane formation. Common characteristics of this group are the association of muscles, brain and eye anomalies. 3 main phenotypes, all with autosomal recessive transmission, have been described within this group: Fukuyama congenital muscular dystrophy (FCMD), muscle eye brain (MEB) and Walker-Warburg phenotype (WWP) or syndrome (WWS) [1]. Among the above, WWP is the most severe and lethal form. Affected individuals are hypotonic at birth and present with severe cerebral and ocular abnormalities.

So far, prenatal diagnosis of this congenital malformation was considered possible only during late pregnancy. Advancements in ultrasound equipment and technology, molecular genetics and histopathology have made very early detection of this syndrome possible, thus shedding new light on the natural history of this entity.

\section{Materials and Methods \\ $\nabla$}

We describe for the first time a very early manifestation of Walker-Warburg phenotype (WWP) using a $6-12 \mathrm{MHz}$ high-resolution transvaginal ultrasound probe (GE Voluson 8) at 11 weeks of gestation confirmed by molecular genetics, postabortion T2 MR imaging (1.5T GE) and histopathology.

\section{Results}

The parents who are a non-consanguineous couple of Ashkenazi Jewish origin were found to carry a founder mutation for Walker-Warburg syndrome in the Fukutin gene as previously described in this ethnic group. This mutation was found following a previous pregnancy that was terminated due to Dandy Walker malformation and cataract. In the present pregnancy chorionic villus sampling (CVS) was scheduled for 11 weeks and 3 days. Transvaginal ultrasound was performed before CVS at 11 weeks to try to characterize possible brain and eye abnormalities at a very early age of gestation. 
A fetus with a CRL of $45 \mathrm{~mm}$ was identified using high-resolution transvaginal ultrasound (TVS 6-12 MHz GE Voluson E8). Innovatively, detailed cranial examination depicted various brain and eye features of WWS at a very early stage of pregnancy. These findings included an enlarged $4^{\text {th }}$ ventricle, high insertion of the tentorium, a prominent lateral ventricle, and a malformed midbrain characterized by the typical 2 kinks within the pons. In addition, ocular findings included cataract of the right lens and smaller left orbit ( $\bullet$ Fig. 1 ).

Molecular analysis of the DNA obtained by CVS revealed a homozygous mutation in the Fukutin gene (FKTN) c.1167_1168insA (p.F390fs) typical for Ashkenazi origin which confirmed the diagnosis ( $\bullet$ Fig. 2 ).

Following dilatation and evacuation (D\&E) at 12 weeks of gestation, we were able to collect the fetal head for further evaluation. The biparietal diameter of the head was $13.6 \mathrm{~mm}$ and post-abortion magnetic resonance imaging (MRI) was performed and showed an enlarged cisterna magna (CM) with asymmetry of the orbits ( $\bullet$ Fig. 3). Histopathology of the fetal cortex revealed changes compatible with cobblestone lissencephaly (๑ Fig. 4).

\section{Discussion}

$\nabla$

This is the first report describing fetal ultrasonographic diagnosis of Walker-Warburg phenotype (WWP) at a very early age of gestation with confirmation of the specific causative genetic mutation.

WWP is a severe lethal malformation manifested by brain and ocular abnormalities: severe hydrocephaly, cobblestone lissencephaly, cerebellar hypoplasia, and posterior encephalocele. Ocular abnormalities include microphthalmia, cataract, and retinal dysplasia $[2,3]$.

In the last 2 decades the routine use of fetal imaging studies enabled prenatal visualization of some of the characteristics of WWP, particularly in patients at risk due to previous affected siblings in the family $[4,5]$.

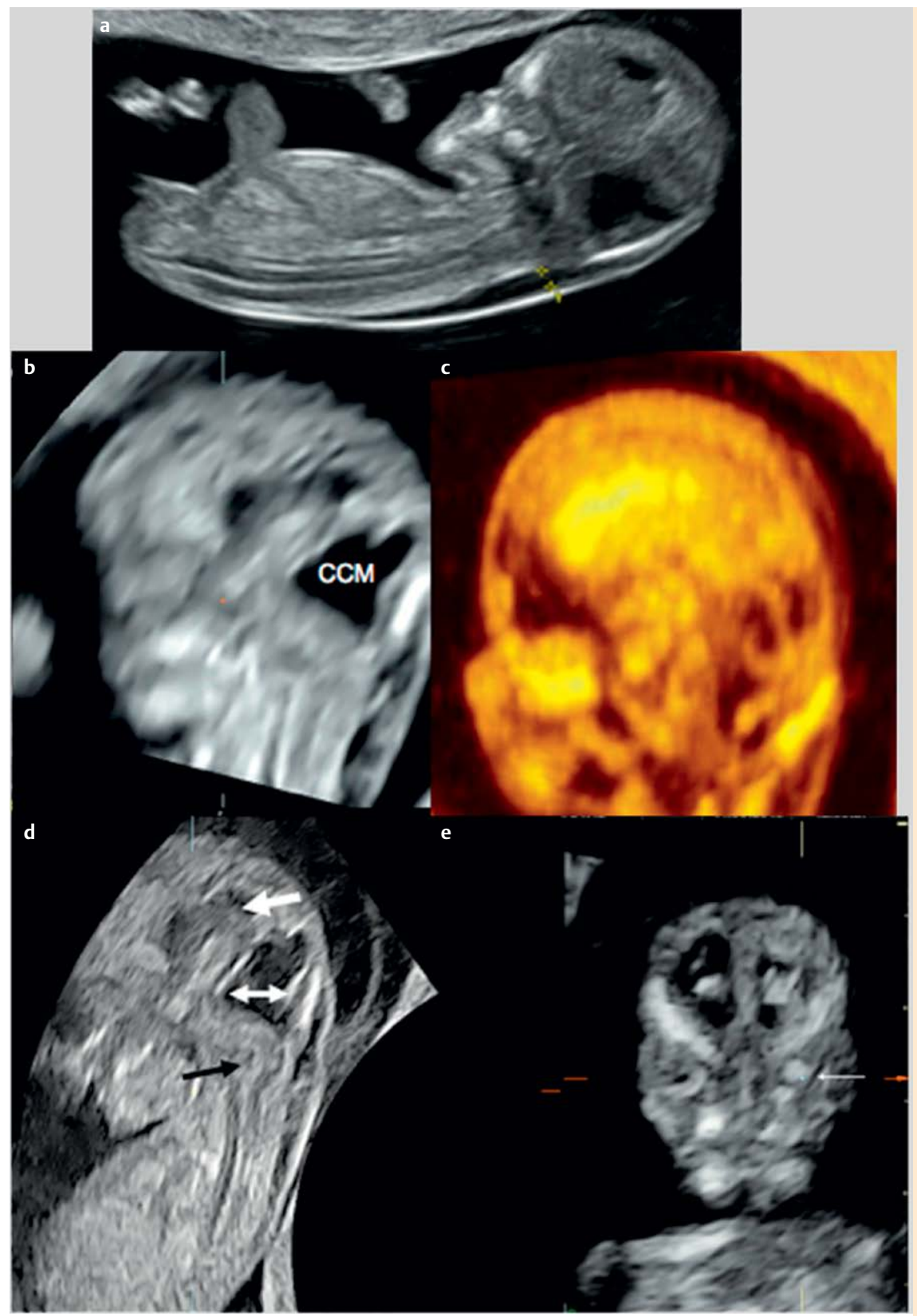

Fig. 1 High-resolution transvaginal sonography of Walker-Warburg phenotype at 11 weeks of gestation with CRL of $45 \mathrm{~mm}$ a. Note the abnormal cystic cisterna magna (CCM), zooming of the region depicting the CCM $\mathbf{b}$ compared to the normal case at the same age c Typical 2 "kink" of the brainstem with large tectum $\mathbf{d}$ hyperechoic lens indicating left eye cataract $\mathbf{e}$. 


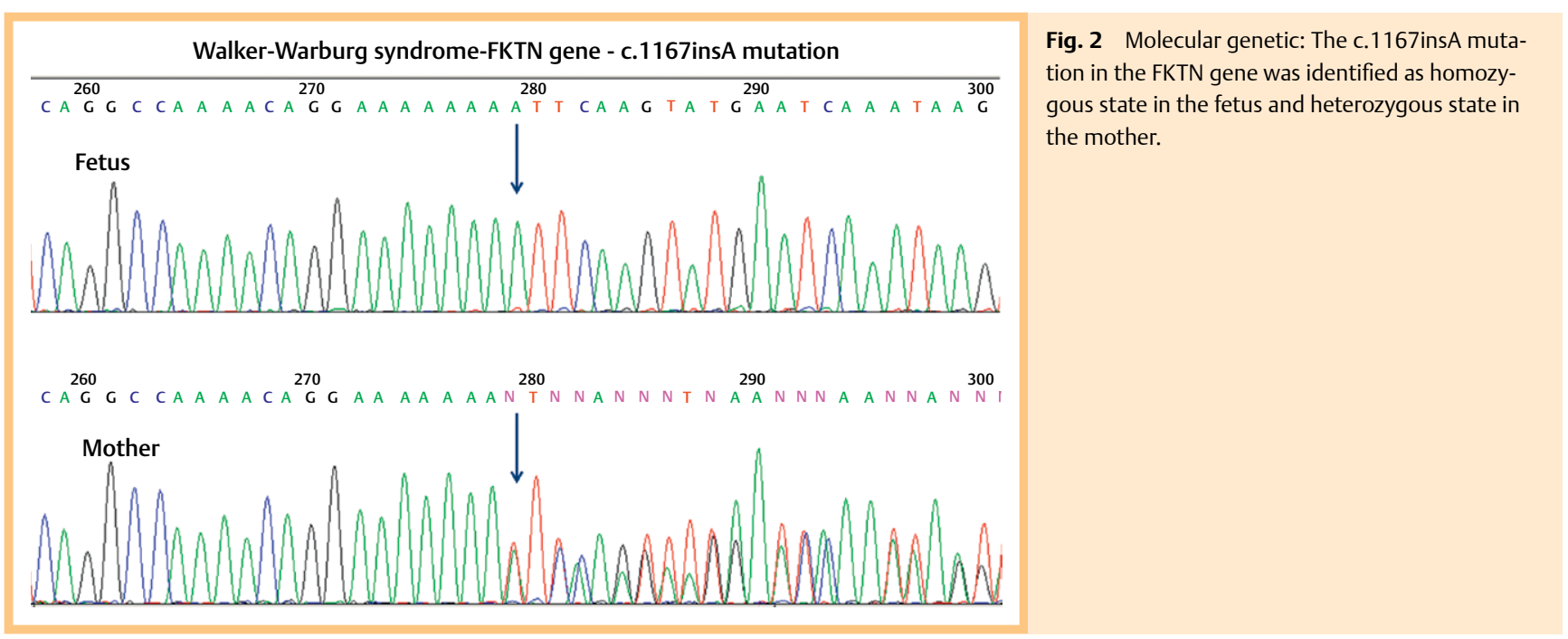

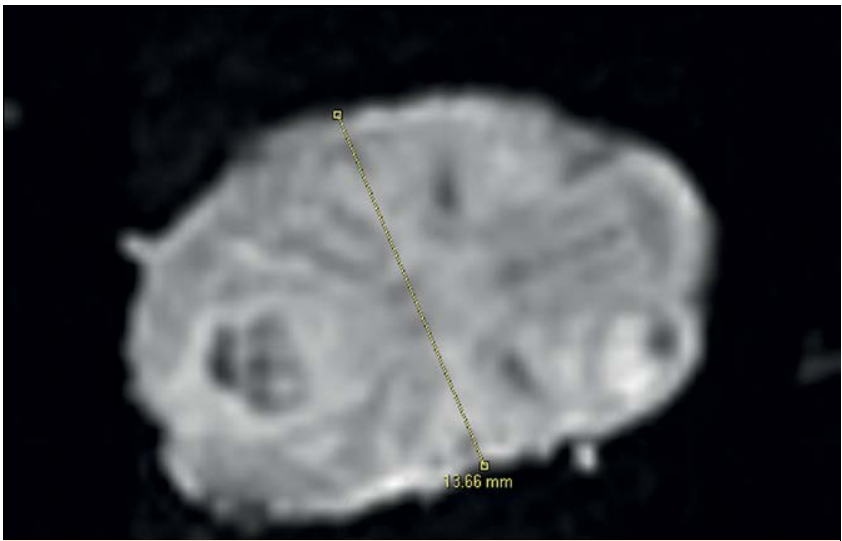

Fig. 3 Post-abortion axial T2-weighted brain MRI showing enlarged cisterna magna (CM) with asymmetry of the orbits. Note that the BPD is $13.6 \mathrm{~mm}$.

Hydrocephaly, which is usually the hallmark for the diagnosis of WWS, appears on the prenatal ultrasound mostly in the third trimester [6]. Although ventriculomegaly has been reported early in gestation between 13-15 weeks, this finding was considered non-specific and therefore a more definitive diagnosis could not have been achieved until late in gestation $[7,8]$.

With the widespread use of fetal MRI, brain imaging studies may reveal a disorganized cortex, shallow gyri (lissencephaly-previous type II), dysplastic cerebellum and characteristic brainstem anomalies.

The typical findings in the brainstem that were described by MRI include pontine hypoplasia, an enlarged quadrigeminal plate with fusion of the superior and inferior colliculi and a distinctive "kink" in the dorsal pons. A second "kink" may be present at the ventral cervicomedullary junction, forming the typical z-shape appearance of the brainstem. However, fetal MRI is applicable mainly late in gestation usually after 28 weeks in the third trimester [9].

Accordingly, ocular anomalies, such as hyperplastic primary vitreous, retinal detachment and dysplasia, which are typical in WWP, have been anecdotally reported at an advance stage of gestation, thus preventing early counseling and management [10-12].
A previous case report in 2005 [6] described a prominent hindbrain vesicle at 12 weeks and dilatation of the lateral ventricle at 14 weeks of gestation, while hydrocephaly and lissencephaly appeared only at 30 weeks. Amniocentesis was performed but without genetic diagnosis since at that time molecular diagnosis was not available [8].

WWP presents with a relatively homogenous phenotype, but it is genetically heterogeneous. The most common etiologies of WWP are mutations in 2 glycosyltransferase, protein O-mannosyltransferase 1 and 2 (POMT1 and POMT2). POMT1 and POMT2 attach the first sugar in the O-mannose-linked glycan moiety of the $\alpha$-dystroglycan protein, a transmembrane glycoprotein expressed on the surface of muscle cells and neurons. B1-dystroglycan protein interacts with several extracellular matrix components in the basal membrane, and disruption of its function is thought to underlie the severe defects in muscle, eye and brain development in WWP. However, mutations in POMT1 were identified in $7-20 \%$ of WWP cases and mutations in POMT2 were found only in $7 \%$ of patients. Mutations in POMGNT1, FKRP LARGE and FKTN have been reported at much lower frequencies among WWP patients [13].

Manzini et al. [14] analyzed the most frequent mutations in 43 individuals with WWP. They observed striking differences in the geographic distribution of mutations. Notably, a specific mutation was found in the FKTN gene in all 3 individuals of Jewish Ashkenazi origin with WWP. Furthermore, a carrier rate of $0.7 \%$ for the (FKTN) c.1167_1168insA (p.F390fs) mutation was found in Ashkenazi Jews in Israel, suggesting a founder effect in this specific population.

Chang et al. [15] identified the same FKTN mutation in 4 families with WWS and also among 2 of 229 normal American Ashkenazi Jewish adults $(0.7 \%)$, thus confirming the previous report.

In the present case we used cutting edge prenatal diagnostic tools (imaging, genetics and pathology) in order to provide a diagnosis very early in pregnancy.

The transvaginal ultrasound performed prior to CVS at 11 weeks of gestation revealed significant features consistent with WWP. The brain findings of prominent lateral ventricles, a giant cisterna magna and the double kink of the mesencephalic-pontine junction are typical for WWP. In addition, the ocular finding of orbital asymmetry with unilateral cataract strongly suggests a fetus affected by WWP. All these anomalies are usually described on fetal MRI in the third trimester. 


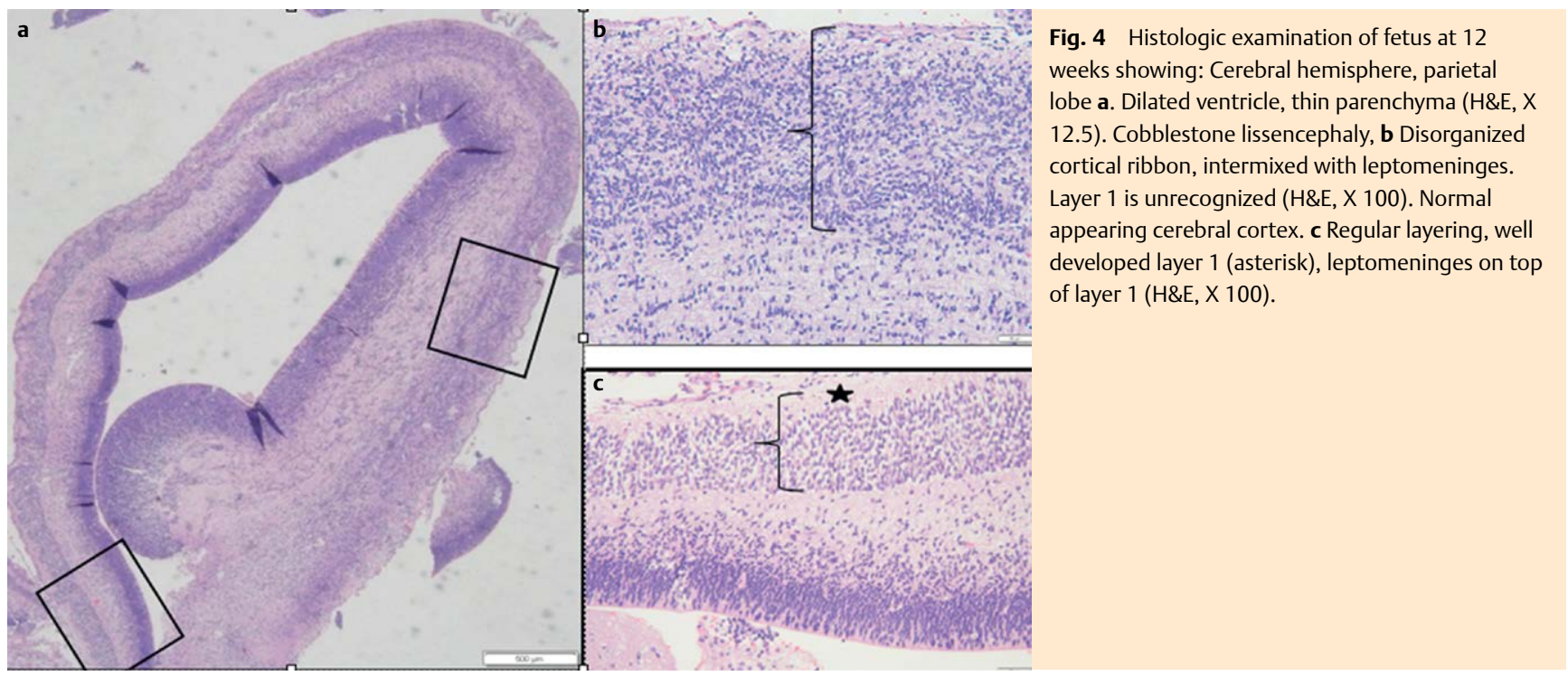

We have shown that the posterior fossa abnormalities appear first, while dilatation of the lateral ventricles which progresses to overt hydrocephalus is a secondarily developing process. Similarly, we have noticed that the anomalies of the eye may start with a smaller orbit and cataract early at gestation, while the retinal detachment and HPVP may appear later on $[8,11]$.

We have already shown that Dandy Walker malformation and eye development could be imaged at a very early gestational age $[16,17]$. Therefore, it is not surprising that we were able to describe these typical findings for WWP so early in gestation.

We have shown that with appropriate preparation we can obtain a good specimen of a small fetal head for further evaluation even when $\mathrm{D} \& \mathrm{E}$ is performed in early gestation.

Indeed this is the first report of using MRI on the product of abortion at 12 weeks gestation and the first to describe the microscopic cortical abnormalities in a fetus with WWP. Additionally, the specific molecular genetics evaluation of the FKTN enabled definitive diagnosis. All the above information led to early decision making before most of the typical signs usually appear. This is a great advantage for patients who wish to terminate pregnancy long before fetal viability when it is still possible according to local law.

Our case is unique since it describes very early changes in the brain and the eyes in a fetus with a priori risk of having WWP. Thus it sheds some light on the time sequence of the appearance of the anomalies in this particular genetic disease.

We show that close collaboration between obstetricians, geneticists and pediatric radiologists improve our ability to manage complicated cases for the benefit of our patients. A thorough search for the molecular basis of this syndrome should follow any fetal brain and eye anomalies.

In addition, the FKTN mutation should be added to the genetic screening programs offered to the Jewish Ashkenazi population.
3 Barkovich JE, Raybaud C. In Pediatric Neuroimaging. 5th EdsLippincott: Williams\&Wilkins (LWW); 2011: 415-416

4 Rodgers BL, Vanner LV, Pai GS et al. Walker-Warburg syndrome: report of three affected sibs. Am J Med Genet 1994; 49: 198-201

5 Gasser B, Lindner $V$, Dreyfus $M$ et al. Prenatal diagnosis of WalkerWarburg syndrome in three sibs. Am J Med Genet 1998; 76: 107-110

6 Low ASC, Lee LS, Tan D et al. Difficulties with prenatal diagnosis of the Walker-Warburg Syndrome. ActaRadiol 2005; 46: 645-651

7 Monteagudo A, Alayón A, Mayberry P. Walker-Warburg Syndrome Case Report and Review of the Literature. J Ultrasound Med 20: 419-426 2001

8 Blin G, Rabbe A, Ansquer $Y$ et al. First trimester ultrasound diagnosis in recurrent case of Walker-Warburg syndrome. Ultrasound ObstetGynecol 2005; 26: 297-299

9 Strigini F, Valleriani A, Cecchi $M$ et al. Prenatal ultrasound and magnetic resonance imaging features in a fetus with Walker-Warburg syndrome. Ultrasound Obstet Gynecol 2009; 33: 363-365

10 Chitayat $D$, Toi A, Babul $R$ et al. Prenatal diagnosis of retinal nonattachment in the Walker-Warburg syndrome. Am J Med Genet 1995; 56: 351-358

11 Yazicioglu HF, Ocak Z. Walker Warburg syndrome with persistent hypoplastic primary vitreous detected by prenatal ultrasonography. Ultrasound Obstet Gynecol 2010; 35: 246-249

12 Brasseur-Daudruy M, Vivier PH, Ickowicz $V$ et al. Walker-Warburg syndrome diagnosed by findings of typical ocular. abnormalities on prenatal ultrasound. Pediatr Radiol 2012; 42: 488-90

13 Yoshioka $M$. Phenotypic spectrum of Fukutinopathy: Most severe phenotype of Fukutinopathy. Brain Development 2009; 31: 419-422

14 Manzini MC, Gleason D, Chang BS et al. Ethnically diverse causes of Walker-Warburg syndrome (WWS): FCMD mutations are a more common cause of WWS outside of the Middle East. Hum Mutat 2008; 29: E231-E241

15 Chang W, Winder TL, LeDuc CA et al. Founder Fukutin mutation causes Walker-Warburg syndrome in four Ashkenazi Jewish families. Prenat Diagn 2009; 29: 560-569

16 Achiron $R$, Achiron A. Transvaginal ultrasonic assessment of the early fetal brain. Ultrasound Obstet Gynecol 1991; 1: 336-344

17 Achiron R, Gottlieb Z, Yaron Y et al. The development of the fetal eye: in utero ultrasonographicmeasurements of the vitreous and lens. Prenat Diagn 1995; 15: 155-60

\section{References}

1 Dobyns WB, Kirkpatrick JB, Hittner HM et al. Syndromes with lissencephaly. II: Walker- Warburg and cerebro-oculo-muscular syndromes and a new syndrome with type II lissencephaly. Am J Med Genet 1985; 22: 157-195

2 Dobyns WB, Pagon RA, Armstrong $D$ et al. Diagnostic criteria for Walker-Warburg Syndrome. Am J Med Genet 1987; 32: 195-210 